\title{
Exploring the Psychosocial Consequences of Mandatory Quarantine During the COVID-19 Pandemic in Hong Kong
}

\author{
Judith Blaine \\ Department of Psychology, Rhodes University, Makhanda, South Africa \\ Email address: \\ j.blaine@ru.ac.za \\ To cite this article: \\ Judith Blaine. Exploring the Psychosocial Consequences of Mandatory Quarantine During the COVID-19 Pandemic in Hong Kong. \\ Psychology and Behavioral Sciences. Vol. 10, No. 2, 2021, pp. 96-103. doi: 10.11648/j.pbs.20211002.13
}

Received: April 24, 2021; Accepted: May 11, 2021; Published: May 20, 2021

\begin{abstract}
Background: While global media and local \& international health organisations provide regularly updated information and statistics on the number of COVID-19 cases and deaths, little is known of the psychosocial impact of COVID-19 quarantine. Quarantine measures during pandemics such as COVID-19 present complex challenges. Determining equitable and effective application of policies is difficult, particularly concerning associated mental health effects. Recent research suggests that as most of the adverse psychological outcomes result from longer quarantine duration and the restriction of liberty, policy makers should consider voluntary quarantine, emphasising altruistic reasons for self-isolating. Having a sense of altruism could mitigate the mental health consequences of quarantine. Objective: The aim of this study was to understand the psychosocial consequences of mandatory quarantine in Hong Kong during the COVID-19 pandemic. Method: An exploratory qualitative research design was employed. Qualitative data from semi-structured interviews and surveys were analysed using template analysis. Findings: Employing the acronym ALTRUISM, the findings suggest that mandatory quarantine was associated with negative psychosocial consequences, some of which were long-lasting. The findings suggest that relying on altruism and not enforcing quarantine could alleviate the mental health issues associated with quarantine. Additionally, psychological support should be made available for people during and after quarantine. Implications: The importance of the contribution of this study to public health policy and practice in Hong Kong and internationally is discussed, with recommendations for future research and practical implications of the findings.
\end{abstract}

Keywords: Quarantine, Psychosocial Consequences, COVID-19, Altruism

\section{Introduction}

Hong Kong (HK) reported their first case of the coronavirus disease 2019 (COVID-19) on 23rd January 2020 and since then widespread measures have been introduced to reduce the spread of the virus: social distancing, compulsory mask wearing, cross-border travel bans and mandatory quarantine regulations. HK has not experienced the mass lockdowns that have taken place in other countries, however, those tested COVID-19 positive are hospitalised and those deemed to be close contacts are placed in mandatory government quarantine camp sites for 14 days. Meanwhile, mandatory quarantine measures for inbound residents have become increasingly stringent: from the initial 14 days home quarantine (March 2020) to 14 days hotel quarantine (October 2020) to the current
Designated Quarantine Hotel Scheme which has been in place since 25 December 2020, requiring all those arriving from countries (other than China, Taiwan \& Macao) to undergo compulsory quarantine for 21 days at designated quarantine hotels [1]. Interestingly, WHO recommends a 14-day quarantine from last exposure to a confirmed case [2]. This is based upon the research showing that the incubation period of COVID-19 can be as long as 14-days, but it is on average 5-6 days [3].

Appreciating that governments, public health authorities and policy makers have to make difficult decisions during a pandemic, WHO guidelines suggest that before governments implement quarantine, they should communicate why this measure is needed, provide clear, up-to-date and consistent guidance about quarantine measures and constructive engagement with communities, if these measures are to be 
accepted. Additionally, those quarantined need access to health care as well as financial, social and psychological support [2].

Along these lines, a number of countries (e.g. New Zealand, Canada, United Kingdom) have devised summary statements based on Thompson, Faith, Gibson and Upshur's [4] ethical framework for pandemic influenza planning [5]. These ethical values, inform how and what decisions are made (please see table 1).

Table 1. Ethical framework to guide decision-making during pandemics. Adapted from Thompson et al [4].

\begin{tabular}{ll}
\hline Ethical Value & Associated Actions \\
\hline Inclusiveness & $\begin{array}{l}\text { To include those who will be affected by the decision, taking into account people from all cultures and communities, taking all } \\
\text { stakeholders contributions seriously and aiming for acceptance of an agreed decision-making process. }\end{array}$ \\
$\begin{array}{l}\text { Openness and } \\
\text { Decisions should be open to scrutiny and the basis for decisions is explained. Information should be effectively disseminated to all } \\
\text { stakeholders and these parties should be clearly informed where to go for further communication }\end{array}$ \\
Reasonableness & $\begin{array}{l}\text { Decisions are based on evidence, principles, and values that are agreed upon and made by those who are credible and accountable. } \\
\text { Responsiveness }\end{array}$ \\
$\begin{array}{l}\text { Decisions should be revisited and revised as new information emerges. } \\
\text { Accountability }\end{array}$ & Ensure mechanisms are in place to guarantee that ethical decision-making is sustained \\
\hline
\end{tabular}

Barbisch, Koenig, \& Shih [6] maintain that those responsible for political decisions to impose quarantine measures should consider evidence-based data prior to restricting civil liberties; while Cheung and Ip [7] state that from a public mental health perspective, extreme quarantine measures should be justified even if they are effective in saving lives. Likewise, Rubin and Wessley [8] argue that while there are epidemiological benefits to humanity of mandatory quarantining, the psychological costs should be taken into account and alternatives should be considered. Chen et al's, [9] recent study emphasised the need to assess the psychological state of, and provide psychological support for, those undergoing quarantine.

Quarantine measures present complex challenges. Determining equitable and effective application of policies is difficult, particularly regarding associated issues of personal liberties. While global media and local \& international health organisations provide regularly updated information and statistics on the number of COVID-19 cases and deaths, little is known of the psychosocial consequences of those in quarantine. Research of pandemics such as SARS, MERS, Ebola and H1N1 has shown that the psychosocial consequences of quarantine are not limited to the fear of contracting the virus [6,10-13]. Other aspects such as loss of freedom, fear of separation from loved ones, uncertainty and powerlessness impact a greater proportion of the population [14].

Recent reviews $[15,16]$ propose that those undergoing quarantine are at an increased risk of negative psychosocial outcomes. Other studies identified a range of psychological consequences of quarantine including anger [13]; fear [12]; grief [17]; numbness [18]; confusion [19]; insomnia, depression and anxiety [20] with increased quarantine duration correlating positively to higher levels of anxiety, post-traumatic stress disorder (PTSD), avoidance behaviour and levels of anger [16]. Brooks et al's [15] review suggests that, in addition to poor communication and lack of supplies \& information, most of the adverse psychological effects resulted from longer quarantine duration and the restriction of liberty. The authors recommend that policy makers consider voluntary quarantine, emphasising altruistic reasons for self- isolating. Similarly, Wang, Shi, Que et al [21] suggest that having a sense of altruism could mitigate the mental health consequences of quarantine.

The WHO Director-General stated that "all countries must strike a fine balance between protecting health, preventing economic and social disruption, and respecting human rights" [22]. Alas, anecdotal evidence from print and social media in Hong Kong suggests a lack of common sense, compassion and humanity in dealing with quarantine measures [23-25]. Currently there is little research on the experiences and psychosocial consequences of mandatory quarantine in Hong Kong during the COVID-19 pandemic, which this research seeks to address. It is hoped that the findings may help government agencies and healthcare professionals to support the physical and mental health of those undergoing quarantine, both in Hong Kong and internationally. These results may also help policy makers to understand how to arrange humanistic quarantine measures, incorporating the ethical values framework in their decision-making process.

\section{Methods}

\subsection{Research Design}

This study adopted an exploratory research design to obtain a profound understanding of the psychosocial consequences of mandatory quarantine during the COVID-19 pandemic in Hong Kong, SAR China.

\subsection{Participant Recruitment}

Participants $(\mathrm{N}=131)$ were recruited via social media, in particular Facebook, and snowball sampling. Table 2 provides the characteristics of the survey population. From this pool of participants, a smaller sample $(\mathrm{N}=14)$ was selected via purposive sampling for in-depth semi-structured interviews. This was done to ensure that the sample was representative of the quarantine population in terms of demographics, experiences and opinions. All participants were required to be $18+$ years or older, Hong Kong residents and able to read and write in English. 
Table 2. Characteristics of Survey Population.

\begin{tabular}{lll}
\hline Characteristics & N & \% \\
\hline Age (years) & & \\
$18-29$ & 15 & 11.81 \\
$30-44$ & 32 & 25.20 \\
$45-60$ & 68 & 53.54 \\
$60+$ & 12 & 9.45 \\
Gender & & \\
Female & 94 & 74.02 \\
Male & 33 & 25.98 \\
Number of times in quarantine (in past 12 months) & & \\
One & 63 & 49.61 \\
Two & 42 & 33.07 \\
Three & 11 & 8.66 \\
Four of more & 11 & 8.66 \\
Type of quarantine & & \\
Home & 28 & 22.05 \\
Hotel & 95 & 74.80 \\
Government quarantine & 3 & 2.36 \\
Hospital & 1 & 0.79 \\
Duration of quarantine & & \\
14 days & 59 & 46.46 \\
21 days & 66 & 51.97 \\
Other & 2 & 1.57 \\
Quarantine alone & & \\
Yes & 82 & 64.57 \\
No & 45 & 35.43 \\
\hline
\end{tabular}

\subsection{Ethical Considerations}

Ethical approval was granted through the institutional research ethics board, and informed consent was obtained online from each participant. Survey responses were anonymous and interviews were confidential with interviewee names removed from the data. The data will be stored securely for five years as required, with access limited to the researcher and only used for the purposes of this study.

\subsection{Data Collection and Analysis}

Data collection took place between $22^{\text {nd }}$ February 2021 and $12^{\text {th }}$ March 2021. Data were collected through online surveys and followed up with semi-structured interviews, primarily using Zoom calls. Surveys took approximately 1520 mins to complete and interviews typically ranged between 30-45 minutes. Data from surveys and semi-structured interviews (transcribed verbatim) were analysed using Template Analysis (TA) [26]. Implementing King's [27] procedural steps, coding was undertaken through: defining $a$ priori themes; familiarisation with the data by initial coding; initial template development and application to data; interpretation of findings; quality and reflexivity checks and producing the report. In TA, because a research project usually begins with an assumption that there are certain aspects of the phenomena that should be focused on, it is quite common to identify themes in advance. Thompson, Faith, Gibson and Upshur's [4] framework of ethical values for decision making (see Table 1) during a pandemic formed the basis of this study's a priori themes (i.e. openness \& transparency, inclusiveness, reasonableness and responsiveness.)

\subsection{Reflexivity}

It has been suggested that reflexivity, as an inter-subjective reflection, forms an important component in improving the rigor and trustworthiness of qualitative research [28]. This refers to the researcher reflecting upon what influences may have had an effect on their interpretation of the data and whether their personal background, beliefs, values and biases could have affected the design, collection and interpretation of the data [29]. The researcher needed to be aware of their own opinions, preconceived ideas and assumptions as well as recognise the role of their own reflections on the findings. Having conducted extensive research on the subject, the researcher attempted to bracket their own knowledge and experience of the subject and, even though it is not ever fully possible, to allow the findings to emerge from the data. Incorporating TA, particularly the a priori code, was useful in this regard as it encouraged a certain amount of structure from previous research findings, whilst allowing flexibility in adapting the codes as other themes/codes emerged from the data.

\section{Quarantine Study Findings}

As expected in qualitative research, prevailing political ideologies and individual differences resulted in different opinions and experiences, from those who believe that COVID-19 is merely a conspiracy theory, to those who feel that mandatory quarantine is a social responsibility. For a very few participants, the experience was actually positive, which they attributed to entering quarantine with a positive mindset. However, for the majority, there was a profound sense of isolation, loneliness, confinement and financial concern.

Thompson, Faith, Gibson and Upshur's [4] ethical framework (Table 1) provided the a priori themes, which assisted in speeding up the initial coding of analysis. The initial template was modified after familiarisation with the data and the apt acronym ALTRUISM was adopted. In what follows, each of the elements of the acronym will be explored with references both to the evidence in the data, as well as links made to the literature.

Key themes explained:

Altruism

Lingering effects of quarantine

Trust

Reasonableness

Unsurprising emotional effects

Inclusivity

Silver linings

Mitigating Mental Health Issues

\subsection{Altruism}

Altruism refers to any behaviour designed to increase the welfare of others, particularly actions that do not appear to provide a direct reward to those performing them [30]. Altruistic or prosocial behaviours are important to ensure that social groups survive and thrive, particularly in times of a pandemic. Blendon et al's [11] study exploring attitudes 
towards quarantine conducted after the SARS epidemic, found that $81 \%$ of Hong Kong respondents favoured quarantining of those suspected of having being exposed to the disease, although only 54\% remained in favour for mandatory quarantine. This supports Wang et al's [21] suggestion that having a sense of altruism could mitigate the negative impact of quarantine on mental health.

Several participants opted to quarantine in hotels rather than put their family at risk by quarantining at home, with one interviewee stating that "I think even if I was given a choice, I would choose to be quarantined in a hotel. Again, just because I have children...". This implies that if people were given a choice, they would most likely use logic and/or altruism and voluntarily quarantine. This prosocial behaviour was echoed in the survey, with one respondent referring to quarantine as a social responsibility "to make sure I am Covid -free to leave the hotel. Knowing others will do the same too". The findings in this study suggest that most people are conscientious towards public health and are compliant with regulations, although there are the few who feel they are above the law and thus alter the status quo for the community.

There was also evidence of altruism in an interviewee who was participating in the hospital COVID study stating that "... for the greater good of man, I've got no problem with doing a trial." Altruism was particularly evident in an online group, the HK quarantine support group, where members delivered groceries, gave advice and provided support for one another at no cost (see mitigating mental health issues).

\subsection{Lingering Effects of Quarantine}

While some participants experienced no long-lasting effects of quarantine, many mentioned lingering physical effects such as back problems, muscle ache and brain fog but more common were lingering feelings of fear, anxiety, anger, exhaustion, lack of energy and difficulty in social integration. Consistent with previous research [13] a number of participants are still experiencing PTSD, depression and insomnia after their quarantine experience, while others feel anger and resentment at the policy makers for imposing this 'punishment' on them. This was evident from the following responses:

a. I think it was the trauma somehow. Like it was a punishment

b. A pathological hatred of the government for locking us up 24/7

c. Resentment at the government policy makers

d. I find it difficult to tell others much about the memory as I feel I have suppressed the memory

One interviewee revealed how their mind blanked out "...my first two weeks, I can't actually remember what I was doing. I genuinely cannot remember." For some participants, the trauma of quarantine was profound and long-lasting with a number of participants requiring medication and therapy for mental health issues. For one interviewee who was hospitalised, the effects were overwhelming: "By the time I got out of hospital, I was so psychologically scarred and terrified, I didn't want to go anywhere. I didn't want to go anywhere where anybody could potentially pick me up and take me back again."

There were some participants who expressed a lingering sense of gratitude for previously taken-for-granted things such as freedom, food, fresh air and friendship following their quarantine experience. However, for the most part, it seemed that it was the lack of openness and transparency that created adverse psychosocial effects.

\subsection{Transparency and Openness}

Although the framework for pandemic planning suggests that decisions should be open to scrutiny and the basis for these decisions explained [5], participants felt that there was a lack of openness and transparency around HK quarantine measures. This lack of transparency and openness led to participants seeking information elsewhere, citing evidence to support conspiracy theories.

One respondent stated that it felt as though the quarantine measures were "designed to deter people from travelling and punish them for doing so" while there were numerous requests that public health authorities provide a rationale for the quarantine measures based on scientific data, particularly the determination that quarantine last 21-days.

a. Firstly, follow the science...21 days quarantine is completely unnecessary.

b. 21 days quarantine is not substantiated. Use data to confirm the need.

These findings correspond with those of Brooks et al [15] who suggest that restricting the length of quarantine, based upon the scientific data and incubation period, could mitigate some mental health effects of quarantine.

Participants felt that while there was some information on the government website, it was not effectively disseminated, with many relying heavily on the HK Quarantine Support Group for information. Moreover, the constantly changing regulations left people feeling uncertain and powerless, resulting in many losing trust in the government. One interviewee stated that

"I think the government has really lost its legitimacy in completely failing to rationalise what it's doing here."

A lack of trust in the authorities affects how people process and interpret health messages and advice. Transparency and honest communication not only mitigates the adverse psychosocial effects of quarantine but will also result in a greater likelihood that the public will accept and adhere to the recommendations made, particularly if reasonable and if they (the public) have been included in the decision-making process [5].

\subsection{Reasonableness}

The ethical value framework purports that decisions should be based on evidence, principles, and values that are agreed upon and made by those who are credible and accountable [4]. While most of the participants supported the need for quarantine measures to reduce the spread of COVID-19, there was a unanimous agreement that 21-days hotel quarantine was unreasonable. This was apparent from 
the survey responses:

a. I don't mind having to quarantine to protect the city and it think it's effective but the third week is just cruel and unnecessary.

b. Quarantine is reasonable, however, given virus incubation period, 21 days hotel quarantine is unnecessary...

A number of participants were more vocal in their survey responses:

a. ...lock people up for 21 days with no scientific basis. It is heinous and likely a violation of the Basic Law

b. 21 days is unnecessary and not supported by data

c. 21 days in a hotel is inhumane

This sentiment was echoed by most of those interviewed, with one interviewee questioning the need for quarantine after being tested negative... "I felt really felt angry... we tested negative, we have no symptoms, why are we being locked up? What are they achieving by doing this? Who are they protecting?" Concerns that these 'draconian' measures are "not connected to real science" have left them feeling afraid.

The general consensus was that home quarantine for 14 days was reasonable and for those for whom this was not feasible, hotel quarantine for 14 days (with a tracking bracelet) would suffice. Several participants that quarantined at home stated categorically that they would not mentally cope with hotel quarantine or hospitalisation, while for many, quarantining at home, in a familiar space, gave them the opportunity to do chores and relax without guilt.

Although only a few participants in this study underwent quarantine in the government quarantine camps, the experience was "hellish" for many [23], with reports of children as young as 2 years old being separated from their families. These stringent measures are bound to have adverse effects on mental health as previous research has suggested [16].

\subsection{Unsurprising Emotional Effects}

In order to glean an overall understanding of the participants experiences of quarantine, a word cloud was generated from the survey responses. The most frequently used words are depicted bolder and bigger (please see figure 1).



Figure 1. Word cloud illustrating participants' response to quarantine.
What is apparent from these responses is a sense of isolation, loneliness, confinement, anxiety, anger, lack of fresh-air and exercise, poor food and financial concerns. The following are just few of the comments from the survey responses:

a. Feeling lonely. The feeling of incarceration

b. Solitary confinement in a strange environment feels like a punishment

c. Feeling of isolation. The financial cost that was unnecessary

d. Being locked up against my will when I've done nothing wrong

These feelings were further endorsed in the interviews suggesting that: "It's a shocking process of isolation where there is no physical contact, and all the windows don't open. It can't be healthy for anyone whatsoever. So you actually think to yourself that this is a punishment. I haven't actually done anything wrong." For many, the fear of 21-days in a hotel or being sent to government quarantine was greater than the fear of getting COVID-19. This was evident in a number of survey responses and interviews as expressed here: "the issue is also, I am personally not so much worried about the disease as such, but the consequences with everybody you have contact with is sent to quarantine which is like a prison."

Financial issues appeared to be a problem particularly the expectation that residents cover the cost of a compulsory 21day hotel stay (and for some coming from 'high-risk' countries, the cost of an additional 21-days in a low-risk country prior to arrival in HK). Several participants mentioned financial anxiety, while one interviewee explained that what was spent for two weeks in a hotel was almost two months of their rent. Respondents suggested that the quarantine experience was directly proportional to the amount of money spent on hotel rooms. This meant that many residents, who had to travel for various reasons, but are unable to afford decent hotels are then made to "suffer 2-3 weeks in subpar conditions." This highlights the inequality of the process and raises the question of how inclusive these measures are for many members of the community.

\subsection{Inclusivity}

Thompson, Faith, Gibson and Upshur's [4] ethical framework advises that all those who will be affected by the measures should be included in the decision-making process, taking into account people from all cultures and communities, and that an acceptance of an agreed decisionmaking process should be attempted. Inclusivity was not something felt by participants in this study. In fact, participants referred to feeling as though they were 'criminals', 'undesirables', 'untouchables' and not human. This was clear from many survey responses with one respondent writing "I felt that I wasn't treated as a human being, from the treatment on arrival in the airport to every time government people contacted me, I was treated as a number and nobody take care of my wellbeing, nutrition, etc." 
Moreover, many felt that they were not heard, despite media reports on the stringent measures and conditions of quarantine [23]. Follow-up interviews shared this sentiment suggesting that: "I'm pretty sure the Hong Kong government are seeing the feedback etc., but I don't think they're really taking any action. But I'm pretty sure they're seeing what we're seeing. Even the hotels. They're noticing what we're saying about it, but they are not really taking actions on it."

In addition, many participants felt while some hotels got it right and made their guests feel welcome, others felt that they were not treated with respect. As one interviewee explained "The whole thing about hotel quarantine for 21 days, is they actually treat you like you're a prisoner..." Those that had some connection with their hotel staff experienced less adverse psychological effects than those who were not given any support. This is corroborated by previous research that shows how important feeling integral to society is for mental wellbeing during quarantine [14]. Once again, the HK quarantine support group was instrumental for many to feel included and connected, even if only through a shared narrative.

\subsection{Silver Linings}

Participants were also asked about the best aspects of, and whether there were any silver linings to, their quarantine experience. While most felt there were none, some participants mentioned that quarantine (mostly those that quarantined at home) gave them time to slow down, to enjoy their own company, and to complete low priority tasks. A number of participants mentioned that quarantine was an opportunity for 'me time' and others felt a sense of accomplishment in completing 21-days quarantine. One interviewee was grateful for the 21-days of being able to structure her own time for her studies, while another used this opportunity to give up smoking.

As Brooks et al [15] argue, initiating social networks during quarantine is vital and being unable to do so is associated with anxiety and long-term distress. Numerous participants were grateful for technology, particularly social media to feel connected. One interviewee reflected that “... we are actually very lucky to go through this pandemic at this time in that technology has enabled us to maintain contact". Netflix was a 'go-to' for many too.

While some participants developed a deeper appreciation for fresh air, friends, family and freedom, the most common positive to emerge was the support and the kindness of strangers from the online support group, which was for many an important component in mitigating mental health issues.

\subsection{Mitigating Mental Health Issues}

Participants were asked what their most helpful tool or daily practice was to mitigate mental health issues and the following word cloud illustrates their responses. Evidently a daily routine, meditation, family and friends proved to be useful for most participants.

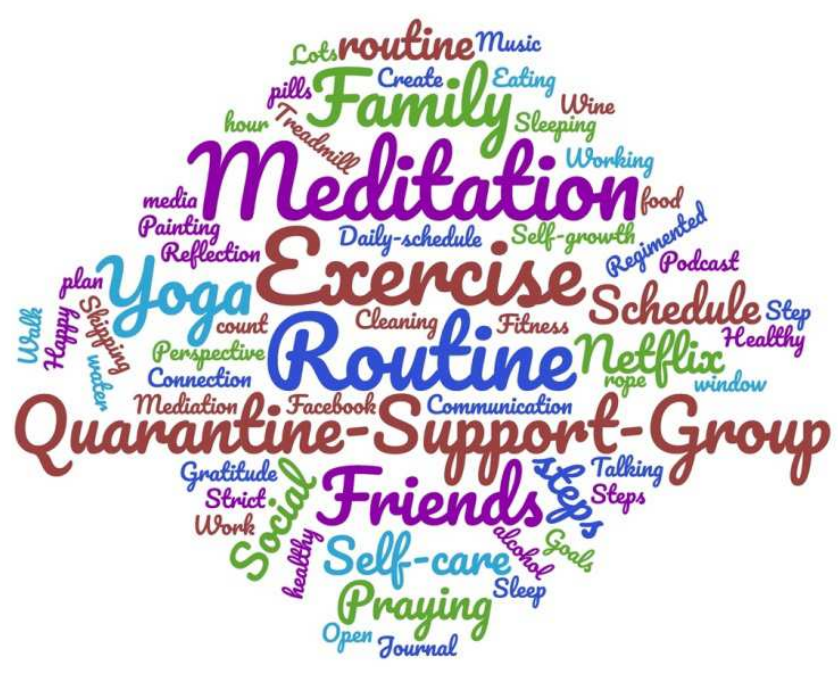

Figure 2. Word cloud illustrating practices used to mitigate mental health issues.

While most participants relied on family and friends for support, the online HK Quarantine Support Group proved to be an incredible source of support for many participants, with an appreciation of the shared narrative of 'incarceration'; many found humour in it too. Members of the group were able to provide guidance and support, with some offering therapy services to members suffering panic attacks and claustrophobia.

Participants acknowledged that while quarantine was bound to have a psychological impact, entering with the positive mindset was paramount to how they coped. One interviewee had a mental wellness coach to help her before entering quarantine and she was able to make the most of the experience. One interviewee who has quarantined several times revealed that they "...learned about the tips I have used to stay sane in quarantine. And by staying sane, I choose those words carefully because to me, so much of the quarantine is a mental battle as much as it is physical."

Participants were asked for suggestions on how policy makers could assist mental wellbeing during quarantine. Aside from the recurring theme of making quarantine shorter and allowing home quarantine, responses included access to fresh air, exercise, decent food and being allowed out once a day. Most participants reported that unless they specifically sought help, there was neither follow up by government officials as to their wellbeing nor recommendations of an active mental health support system with regular checking in for those in quarantine, nor free access to counsellors or psychologists if need be. These recommendations are in line with previous research which suggests professionally staffed telephone support lines providing comfort and social connection for those in quarantine should be available at all hours [15].

\section{Discussion}

Despite the epidemiological benefits of quarantining, it is often associated with negative psychosocial consequences [9, 
12, 13]. In this study, many of those who experienced quarantine expressed feelings of isolation, confinement, loneliness, anger, depression and anxiety, some of which remained even after they returned home. Those that quarantined for 21-days seemed to experience the most adverse psychosocial effects, supporting previous research that showed that longer quarantine is associated with poorer psychological outcomes [15].

Concurring with previous research [10] participants mentioned that poor information and a lack of transparency from the government health officials contributed to their stress, particularly the unsubstantiated rationale regarding the length of quarantine. It is recommended that clear and transparent information on the rationale for decisions made should be provided by the government and policy makers. This concurs with Gray's [5] findings that people are more likely to accept difficult decisions if the decision-making process follows bioethical values (i.e. it is reasonable, open and transparent, inclusive, responsive and accountable).

Additionally, practical advice on coping strategies should be provided and accessibility to mental health professionals for those that require it, should be available during and after quarantine. Although many expressed negative psychosocial effects, most participants adopted positive coping strategies, including external support from an online community. This supports previous research that found belonging to such a group and feeling connected to others in a similar situation could be supportive and empowering [15].

Those that quarantined at home fared better than those in hotels, hospital or government quarantine. Almost all participants appreciated the necessity of quarantine measures and were prepared to voluntarily quarantine at home; it seemed that the mandatory nature of the measures, without scientific backing, evoked negative affect. It would be interesting to explore whether voluntary versus mandatory quarantine has any bearing on psychosocial wellbeing.

Despite this study being the first of its kind to explore the psychosocial consequences of mandatory quarantine in HK, there are several limitations of this study that need to be noted. This sample is restricted to HK quarantine measures and respondents were recruited primarily from the $\mathrm{HK}$ quarantine support group, and as such the experiences expressed may not be representative of the population. These data are preliminary and exploratory and require further replication and investigation. Another limitation is the possibility of the researcher influencing the interviews and data collection due to her own preconceived ideas surrounding quarantine measures.

\section{Conclusion}

This research sheds light on the perspectives and experiences of $\mathrm{HK}$ residents towards quarantine measures that may inform policies in the future. Given the need to balance public safety with human rights, this study highlights the need for inclusiveness, reasonableness, openness and transparency when making decisions regarding quarantine measures in order to mitigate negative psychosocial consequences. Moreover, relying on altruism and not enforcing quarantine would also alleviate the mental health issues associated with quarantine. Additionally, psychological support should be made available for people during and after quarantine. This study has implications for public health policy and practice, in HK and internationally, to arrange humanistic quarantine measures and to incorporate the ethical values framework in their decision-making process.

\section{Declaration of Conflict of Interest}

The author declared no potential conflict of interests with respect to the research, authorship and/or publication of this article.

\section{Funding}

The author received no funding for this research, authorship and/or publication of this article.

\section{Acknowledgements}

The author would like to thank the participants who joined this study. The author acknowledges the ethical approval from Rhodes University.

\section{References}

[1] The Government of HKSAR (Hong Kong Special Administrative Region), (2021). Quarantine for inbound travellers - frequently asked questions. https://www.coronavirus.gov.hk/eng/inbound-travelfaq.html\#FAQ1

[2] World Health Organisation (WHO), (2020). Considerations for quarantine of contacts of COVID-19 cases. https://www.who.int/publications/i/item/considerations-forquarantine-of-individuals-in-the-context-of-containment-forcoronavirus-disease-(covid-19)

[3] Yu, P., Zhu, J., Zhang, Z. \& Han, Y. (2020). A familial cluster of infection associated with the 2019 novel Coronavirus indicating possible person-to-person transmission during the incubation period. Journal of Infectious Diseases. 221 (11): 1757-61.

[4] Thompson, A. K., Faith, K., Gibson, J. L. \& Upshur R. (2006). Pandemic influenza preparedness: an ethical framework to guide decision-making. BMC Med Ethics (7) 12. https://doi.org/10.1186/1472-6939-7-12

[5] Gray, B. (2020). COVID-19 from Wellington New Zealand. Bioethical inquiry $17, \quad 633-638$ https://doi.org/10.1007/s11673-020-10038-3

[6] Barbisch, D., Koenig, K. L., \& Shih, F. Y. (2015). Is there a case for quarantine? Perspectives from SARS to Ebola. Disaster medicine and public health preparedness, 9 (5), 547553. https://doi.org/10.1017/dmp.2015.38 
[7] Cheung, D. \& Ip, E. (2020). COVID-19 lockdowns: a public mental health ethics perspective. $A B R \quad 12, \quad 503-510$ https://doi.org/10.1007/s41649-020-00144-0

[8] Rubin, G. J., \& Wessely, S. (2020). The psychological effects of quarantining a city. BMJ (Clinical research ed.), 368, m313. https://doi.org/10.1136/bmj.m313

[9] Chen, D., Song, F., Tang, L., Zhang, H., Shao, J., Qiu, R., Wang, X. \& Ye, Z. (2020). Quarantine experience of close contacts of COVID-19 patients in China: A qualitative descriptive study. General Hospital Psychiatry. 66. 81-88.

[10] Braunack-Mayer, A., Tooher, R., Collins, J. E., Street, J. M. \& Marshall, H. (2013). Understanding the school community's response to school closures during the H1N1 2009 influenza pandemic. BMC Public Health. 13: 344. 12.

[11] Blendon, R. J., DesRoches, C. M., Cetron, M. S., Benson, J. M., Meinhardt, T., \& Pollard, W. (2006). Attitudes toward the use of quarantine in a public health emergency in four countries. Health affairs (Project Hope), 25 (2), w15-w25 https://doi.org/10.1377/hlthaff.25.w15

[12] Caleo, G., Duncombe, J., Jephcott, F., Lokuge, K., Mills, C., Looijen, E., Theoharaki, F., Kremer, R., Kleijer, K., Squire, J., Lamin, M., Stringer, B., Weiss, H., Culli, D., Di Tanna, G. L., \& Greig, J. (2018). the factors affecting household transmission dynamics and community compliance with Ebola control measures: a mixed-methods study in a rural village in Sierra Leone. BMC Public Health. 18: 248. 13.

[13] Cava, M. A., Fay, K. E., Beanlands, H. J., McCay, E. A., \& Wignall, R. (2005). The experience of quarantine for individuals affected by SARS in Toronto. Public health nursing (Boston, Mass.), 22 (5), 398-406. https://doi.org/10.1111/j.0737-1209.2005.220504

[14] Saladino, V., Algeri, D., \& Auriemma, V. (2020). The psychological and social impact of Covid-19: new perspectives of well-being. Frontiers in psychology, 11, 577684. https://doi.org/10.3389/fpsyg.2020.5776

[15] Brooks, S. K., Webster, R. K., Smith, L. E., Woodland, L., Wessely, S., Greenburg, N., Rubin, G. J. (2020). The psychological impact of quarantine and how to reduce it: rapid review of the evidence. The Lancet. 395: 912-920.

[16] Henssler, J., Stock, F., van Bohemen, J., Walter, H., Heinz, A., \& Brandt, L. (2021). Mental health effects of infection containment strategies: quarantine and isolation-a systematic review and meta-analysis. Eur Arch Psychiatry Clin Neurosci 271, 223-234 https://doi.org/10.1007/s00406-020-01196-x

[17] Wang, Y., Xu, B., Zhao, G., Cao, R., He, X. \& Fu, S. (2011). is quarantine related to immediate negative psychological consequences during the 2009 H1N1 epidemic? Gen Hosp Psychiatry (33) 75-77.

[18] Pan, P., Chang, S-H., \& Yu, Y-Y. (2005). A support group from home-quarantined college students exposed to SARS: learning from practice. J Spec Group Work. 2005; 30: 363375 .

[19] DiGiovanni, C., Conley, J., Chiu, D., \& Zaborski, J. (2004). Factors influencing compliance with quarantine in Toronto during the 2003 SARS outbreak. Biosecurity and bioterrorism: biodefense strategy, practice, and science, 2 (4), 265-272. https://doi.org/10.1089/bsp.2004.2.265

[20] Wang, C., Song, W., Hu, X., Yan, X., Zhang, X., Wang. X \& Chen, W. (2021). Depressive, anxiety, and insomnia symptoms between population in quarantine and general population during the COVID-19 pandemic: a case-controlled study. BMC Psychiatry 21, 99. https://doi.org/10.1186/s12888021-03108-2

[21] Wang, Y., Shi, L., Que, J., Lu, Q., Liu, L., Lu, Z., Xu, Y., Liu, J., Sun, Y., Meng, S., Yuan, K., Ran, M., Lu, L., Bao, Y. \& Shi. J. (2021). The impact of quarantine on mental health status among general population in China during the COVID-19 pandemic. Mol Psychiatry. https://doi.org/10.1038/s41380021-01019-y

[22] World Health Organisation (WHO) (2020b). WHO DirectorGeneral's opening remarks at the mission briefing on COVID19 - 12 March 2020. https://www.who.int/directorgeneral/speeches/detail/who-director-general-s-openingremarks-at-the-mission-briefing-on-covid-19---12-March2020

[23] Vines, S. (2021). Does Hong Kong's quarantine centre need to be quite so hellish? Hong Kong Free Press. https://hongkongfp.com/2021/02/27/steve-vines-does-hongkongs-quarantine-centre-need-to-be-quite-sohellish/?fbclid=IwAR0PIF8V0Hfa1QqojkOVnYJReofyeq32DO6TLqQgeJjFdjOgflaQG2e8Ko

[24] Whitehead, K. (2021, $16^{\text {th }}$ March). In Hong Kong, going into quarantine is more likely than catching COVID-19. South China Morning Post. https://www.scmp.com/magazines/postmagazine/short-reads/article/3126853/hong-kong-goingquarantine-more-likely-catching

[25] Hong Kong hotel quarantine rules: a plea for common sense and mercy. (2021, $9^{\text {th }}$ March). South China Morning Post. https://www.scmp.com/comment/letters/article/3124519/hongkong-hotel-quarantine-rules-plea-common-sense-and-mercy

[26] King, N. (2004). Using Templates in Thematic Analysis of Text. In C. Cassell \& G. Symon (Eds) Essential guide to qualitative methods in organizational research (pp. 256-270). London: Sage.

[27] King, N. (2012). Doing template analysis. In G. Symon \& C. Cassell (Eds). Qualitative Organizational Research: core methods and current challenges (pp. 426-459). London: Sage.

[28] Hollway, W. (2007). Methods and knowledge in social psychology. In W. Hollway, A. Lucey, \& A. Phoenix. (Eds) Social Psychology Matters (pp 84-111). Milton Keynes: Open University Press.

[29] Finlay, L., \& Langdridge, D. (2007). Embodiment. In W. Hollway, H. Lucey, \& A. Phoenix (Eds.) Social Psychology Matters. (pp 190-219). Maidenhead, Open University Press.

[30] Batson, C. D., Ahmad, N., \& Stocks, E. L. (2004). Benefits and liabilities of empathy-induced altruism. In A. Miller (Ed.), The social psychology of good and evil (pp. 359-385). New York: Guilford Press. 